\title{
PERSISTENT BINOCULAR DIPLOPIA FOLLOWING CATARACT SURGERY: AETIOLOGY AND MANAGEMENT
}

\author{
JUDITH WYLIE', MYRTLE HENDERSON', MELANIE DOYLE' and MARIE HICKEY-DWYER ${ }^{2}$ \\ Liverpool
}

\begin{abstract}
SUMMARY
We studied all patients referred to the orthoptic department with binocular diplopia following cataract surgery between January 1991 and June 1993. Persistence of diplopia for a minimum of 3 months after cataract surgery was required for inclusion in the study. Eighty-one patients (2\% of all patients who underwent cataract surgery during this time) satisfied the entry criteria. The patients fell into two groups: non-traumatic and traumatic cataracts. Horizontal deviations were seen in $\mathbf{2 4}$ patients. Vertical deviations were seen in 8 patients and a combined horizontal and vertical deviation was seen in 49 patients. Fresnel prisms were used to manage the diplopia in 58 patients. Of these, 48 patients in the non-traumatic group regained binocular single vision with this prism while 10 in the traumatic group benefited. Mechanical and sensory causes are discussed.
\end{abstract}

The onset of diplopia after otherwise successful cataract surgery is distressing for patients. Many factors may contribute to the occurrence of this problem including prolonged sensory deprivation resulting in disruption of sensory fusion, ${ }^{1-3}$ and paresis of one or more ocular muscles. ${ }^{1.4}$ Other possible aetiologies include myotoxic effects from local anaesthesia ${ }^{5-7}$ or subconjunctival gentamicin injections. ${ }^{8.9}$

Previous studies which have addressed this problem have been on small numbers of selected patients undergoing strabismus surgery or have included patients with dysthyroid eye disease. ${ }^{10.11}$

We report the incidence of diplopia in patients following cataract surgery. We examine the aetiology and recommend management guidelines for this unique group of patients.

\section{PATIENTS AND METHODS}

One hundred and nineteen consecutive patients complain-

From: 'Orthoptic Department and 'Senior Registrar, St Paul's Eye Unit, Royal Liverpool University Hospital, Liverpool, UK.

Correspondence to: Mrs J. Wylie, DBO(D), St Paul's Eye Unit, Royal

Liverpool University Hospital, Prescot Street, Liverpool L7 8XP. UK. ing of diplopia were evaluated in the orthoptic department following cataract surgery. Persistence of binocular diplopia for longer than 3 months following cataract surgery was required for inclusion. Patients who had previously undergone retinal detachment or strabismus surgery were excluded. Patients with a history of dysthyroid eye disease were also excluded.

Each patient had a full ophthalmological and medical history recorded. All patients had a full ophthalmological examination and orthoptic assessment. Eighty-one patients satisfied the entry criteria for the study. Patients fell into two distinct groups: a non-traumatic cataract group and a traumatic cataract group. Table I summarises the patient characteristics.

In the non-traumatic group 36 patients (72 eyes) had bilateral and 33 (33 eyes) unilateral cataract extraction. Forty-four patients (67 eyes) had a general anaesthetic, 16 (20 eyes) had local anaesthetic and 9 (18 eyes) had both. Ninety-five operations were extracapsular extractions, 5 were phacoemulsification, 3 were intracapsular extractions and the remaining 2 were lensectomies. Pre-operative visual acuity ranged from $1 / 60$ or less (36 eyes), to 2/60-6/24 (49 eyes), 6/18-6/12 ( 19 eyes) or 6/9 ( 1 eye).

All patients had a superior rectus bridle suture. Patients undergoing extracapsular extraction had a corneal section and insertion of a posterior chamber lens. They were followed up in the outpatients clinic 1 week, 6 weeks and 3 months post-operatively. The patients were refracted and had their error corrected between 2 and 5 months following surgery.

Of the 12 traumatic cases all had a general anaesthetic

Table I. Study population

\begin{tabular}{lcc}
\hline & \multicolumn{2}{c}{ Subgroups } \\
\cline { 2 - 3 } Patient characteristics & Non-traumatic & Traumatic \\
\hline Total no. of patients & 69 & 12 \\
No. of males & 30 & 12 \\
No. of females & 39 & 0 \\
Age range (years) & $33-95$ & $14-53$ \\
Mean age (years) & $77^{\text {a }}$ & 34 \\
\hline
\end{tabular}

"Three patients younger than 50 were excluded. 
for the cataract extraction and 1 had a posterior chamber intraocular lens inserted at the time of the primary procedure. Two patients had a second procedure to insert a secondary anterior chamber intraocular lens, one under general and one under local anaesthetic. Another had a surgical capsulotomy. Originally 11 and then 9 patients had aphakic correction using a contact lens. Of the patients who had a contact lens fitted the length of time between surgery and fitting varied between 2 and 12 months; the mean was 6 months. Pre-operative visual acuity was 1/60 or less in all 12 patients. No patient in this group had a previous history of diplopia. All patients in both groups had a subconjunctival injection of gentamicin at the end of the surgical procedure.

\section{RESULTS}

The interval between onset of diplopia and cataract surgery varied among patients, occurring either immediately or early in the post-operative period (27 patients), or later at the time of refraction 6-12 weeks post-operatively (29 patients). Six patients complained of diplopia following Yag capsulotomy which was carried out 4-6 months postoperatively. Seven patients were unsure about the timing of its onset.

Nineteen patients were referred to the orthoptic department on the same day as they complained of diplopia at their consultation with the doctor or optician. For the other 50 the timescale was 1-7 months (mean 4 months). In 7 cases there was a delay of 12 months. Post-operative visual acuity ranged from 6/60-6/24 (4 eyes), to 6/18 (10 eyes), 6/12 (13 eyes), 6/9 (44 eyes), 6/6 (21 eyes), 6/5 (12 eyes) or $6 / 4$ ( 1 eye).

Patients in the non-traumatic group had either a horizontal, vertical, or combined horizontal and vertical deviation (Table II). Seventy-one per cent had a vertical element. The muscle weaknesses found were superior rectus in 13 patients ( 12 ipsilateral, 1 contralateral and 1 also had ptosis); inferior rectus in 6 patients (all ipsilateral); superior oblique in 2 patients ( 1 ipsilateral and 1 contralateral); and bilateral lateral recti in 10 patients. The other 38 patients showed no incomitance. Four patients had a tilted intraocular lens. Three of these demonstrated a vertical deviation and one a horizontal deviation. The majority of patients (8) in the traumatic group had a combined exodeviation with a vertical element; 1 also had torsion. Two patients had a vertical deviation due to weakness of the superior rectus muscle in the operated eye. Two patients had a deviation varying between convergent and divergent with a vertical element.

Table II. Deviations in non-traumatic group of patients

\begin{tabular}{lcl}
\hline Type of deviation & No. of patients & Range of deviation (PD) \\
\hline Eso & 10 & $2-25 \mathrm{BO}$ \\
Exo & 10 & $2-20 \mathrm{BI}$ \\
Vertical & 8 & $2-9$ \\
Combined & 7 & $2-14 \mathrm{BO} / 2-9$ \\
$\quad$ Eso/vertical & 34 & $2-25 \mathrm{BI} / 2-14$ \\
$\quad$ Exo/vertical &
\end{tabular}

Exo, exotropia; Eso, esotropia; PD, prism dioptres; BO. base out; BI. base in.
Twelve patients had an orthoptic examination before cataract surgery. Of these, 3 had a change in their deviation which could be attributed to the cataract surgery. In 2 of these a vertical element appeared and both had an underacting superior rectus which was not present before surgery. The horizontal angle changed in the third patient due to a reduction in myopia. Another 3 patients had a change in their deviation which was probably coincidental to their cataract surgery. The remaining 6 had no change in their deviation. All patients were more aware of binocular diplopia following the cataract surgery. Six of these patients had very poor vision in the affected eye prior to surgery.

\section{Binocular Single Vision}

Of the 69 patients in the non-traumatic group $48(70 \%)$ were helped by a Fresnel prism. All these patients achieved binocular single vision (BSV) and stereopsis. Twenty-four patients achieved 200 secs of arc or better. The Fresnel prism required was horizontal in 16, vertical in 21 and combined horizontal and vertical (oblique) in 11 patients. The power of the prism was stable in 29 patients from the time of its first application and changed in 19, either increasing ( 5 patients) over 3-18 months or decreasing ( 12 patients) over $1-12$ months. In 2 patients the prism neither increased nor decreased but changed orientation from being purely vertical to horizontal. The other changes are summarised in Tables III and IV.

Thirteen patients regained BSV without the aid of a prism. Another 2 patients were helped with convergence exercises. In 3 patients the deviation was too large to help with prisms. Two of these have had strabismus surgery. Two patients refused to wear the prism and 1 patient learned to ignore the false image.

Ten patients in the traumatic group first complained of diplopia following aphakic correction with a contact lens. Three were symptomatic on the first day of correction and were referred immediately to the orthoptic department. The patient who had a posterior chamber lens inserted at the primary procedure and 1 of the 2 patients who had a secondary anterior chamber lens inserted complained on the first post-operative day. The remainder were referred between 1 and 9 months, the mean being 4 months. Five patients had a corrected visual acuity of $6 / 60-6 / 18$, and 7 had $6 / 12$ or better.

Ten patients were helped with a Fresnel prism. Two of these patients have been listed for squint surgery although their diplopia remains relieved with a strong prism. In one other the angle of deviation is too large to be helped with a prism. The patient with torsion is partially relieved with a prism but remains symptomatic. Both are awaiting surgery. The patient with the primary posterior chamber lens gained BSV without a prism. The strength of the Fresnel prism in 5 patients changed. One improved over a period

Table III. Non-traumatic group: increase in prism power

\begin{tabular}{lccc}
\hline $\begin{array}{l}\text { Total no. } \\
\text { of patients }\end{array}$ & Horizontal & Vertical & $\begin{array}{c}\text { Horizontal } \\
+ \text { vertical }\end{array}$ \\
\hline 5 & 2 & 2 & 1 \\
\hline
\end{tabular}


Table IV. Non-traumatic group: decrease in prism power

\begin{tabular}{lccc}
\hline $\begin{array}{l}\text { Total no. } \\
\text { of patients }\end{array}$ & Horizontal & Vertical & $\begin{array}{c}\text { Horizontal } \\
+ \text { vertical }\end{array}$ \\
\hline 12 & 5 & 7 & 0 \\
\hline
\end{tabular}

of 8 months resulting in BSV without the aid of a prism. The prism strength in the other 4 is still unstable. One has decided to discontinue wearing the contact lens. One has been lost to follow-up.

\section{DISCUSSION}

The incidence of diplopia following cataract surgery in our series was $2 \%$. There have been no previous studies documenting the incidence of this problem. Although the absence of diplopia before the cataract surgery may implicate operative manipulations or optical aberrations this is not necessarily the case. Our study is not biased in favour of vertical deviations as the patients were referred due to diplopia, not for a motility consultation.

There was no increased incidence of superior or inferior rectus muscle weakness between those patients who had local or general anaesthetic. Therefore direct myotoxicity due to the local anaesthetic is unlikely to have been causative. Other workers have reported a relationship between local anaesthetic and inferior rectus muscle restrictions. $^{6-8,12.13}$ These studies have suggested that such restrictions may be caused by local scar tissue or vascular compromise with extraocular muscle ischaemia. Elevation and depression showed no evidence of restriction in any of our patients. Our findings cannot explain the development of such restrictions. The strength of local anaesthetic was lower in our series than other reports ${ }^{\gamma}$ and perhaps this is the most likely explanation for the few problems seen in our patients secondary to the local anaesthetic.

Superior rectus muscle underaction occurred in 14 cases. Surgical trauma to this muscle due to the bridle fixation suture, or intraoperative traction on the muscle bundles with subsequent muscle contracture, are possible causes. ${ }^{4.10 .14 .15}$ None of our cases demonstrated restriction; therefore the under-action was probably due to limited muscle fibre damage which could be consistent with direct trauma.

Subconjunctival gentamicin has been reported to cause myotoxicity in rabbits ${ }^{9}$ and by Kushner in a patient in 1988 . $^{16}$ This could have been responsible for inferior rectus weakness in our patients although in Kushner's patient the muscle was restricted. The volume of gentamicin used in our patients was $0.5 \mathrm{ml}$. In the subconjunctival space it is unlikely to have exerted sufficient pressure to compress the muscle and is more likely to have been directly toxic to the muscle fibrils as previously reported. To avoid this, the injection should be directed away from the extraocular muscles and we advocate the inferomedial quadrant.

Superior and inferior rectus muscle under-action in our series was stable in $70 \%$ of cases. This is indicative of irreversible but non-progressive muscle damage. Non-progression of vertical deviations has been reported by Burns et al. ${ }^{17}$ in a small series of 4 patients. Those deviations which were unstable all improved.

The deviations were smaller than in other series ${ }^{4.6 .7 .13 .1+.18}$ and were unstable in $49 \%$ of all patients. Instability persisted for an average of 5 months. Previous studies have alluded to this instability but have not linked it with causation. Decompensation of heterophorias due to sensory deprivation is consistent with our findings of small deviations. This would explain the spontaneous improvement in 13 patients and in 6 other patients treated with prisms following surgery. In patients with pure vertical diplopia instability was associated with improvement in all cases and suggests that where surgical trauma is mild, recovery occurs.

Several reports have documented the finding of muscle restrictions either on clinical examination or with forced duction testing. Hamed and Mancuso ${ }^{7}$ suggested that some cases of superior rectus under-action could be attributed to inferior rectus restriction. None of our patients demonstrated this finding on clinical examination of ductions, elevation or depression. It is difficult to explain this difference but it implies severe trauma in the area of the affected muscle.

Brent ${ }^{19}$ studied a group of patients undergoing cataract surgery both pre- and post-operatively and found that post-operative diplopia occurred in $20 \%$ of the patients with a pre-existing tropia. In our series 6 of 12 patients seen pre-operatively had a change in their deviation with subsequent change in their diplopia. In half of these the change could be attributed to the surgery and in the other 3 there was no relationship. Patients with a pre-existing tropia should be informed of the possibility of post-operative diplopia.

Previous studies reporting on diplopia in patients following cataract surgery have concentrated on the motility problems and their management. ${ }^{1.12 .17 .2()}$ Diplopia in the absence of motility disorders has largely been ignored. In our series almost $50 \%$ of the patients had concomitant deviations. The management of these patients and those with muscle under-actions which recovered slowly has not been dealt with in the literature to date. In our study we had the opportunity to do this. We observed and monitored the deviations and tailored the treatment to the patients' changing needs. Deviations were unstable in $49 \%$ of all patients. We found Fresnel prisms were of benefit to 48 patients in the non-traumatic group and 10 in the traumatic group. The relatively small size of deviations in our patients meant that they could have prismatic treatment without visual compromise. These prisms were useful in eliminating diplopia as a temporising measure in the postoperative period whilst patients were either awaiting muscle recovery or regaining binocular single vision. Fresnel prisms were an easy-to-use method of alleviating diplopia as they could be readily changed. We recommend them as a cost-effective way of managing changing diplopia. In our series the diplopia stabilised in the majority of patients between 5 and 7 months post-operatively at which time a prism, if still required, could be incorporated into 
their spectacles. Treatment with Fresnel prisms in many of our patients was definitive. In contrast, other studies use them only as a temporising or diagnostic tool.

In conclusion, diplopia is a risk following cataract surgery for patients with both non-traumatic and traumatic types of cataract. Patients with a pre-existing tropia should be informed of the possibility of post-operative diplopia. In this study muscle deviations were small. Instability was a good prognostic sign, most patients demonstrating reduction in the size of their deviation. The incidence and severity of diplopia following cataract surgery could be reduced by judicious manipulation of the extraocular muscles and instillation of subconjunctival gentamicin into the inferomedial quadrant at the time of surgery. Fresnel prisms as a cost-effective management tool should be the treatment of choice if diplopia does occur.

Key words: Cataract, Diplopia, Fresnel prism.

\section{REFERENCES}

1. Hamed LM, Helveston EM, Ellis FD. Persistent binocular diplopia after cataract surgery. Am J Ophthalmol 1987;103:741-4.

2. Tillson G, Pratt-Johnson JA. Fusion loss from longstanding unilateral cataracts in adults. In: Lenk-Schafer M, editor. Orthoptic horizons. London: British Orthoptic Society, 1988. Transactions of the 6th International Orthoptic Congress, Harrogate, UK, June 29-July 2, 1987.

3. Pratt-Johnson JA, Tillson G. Intractable diplopia after vision restoration in unilateral cataract. Am $\mathbf{J}$ Ophthalmol 1989;107:23-6.

4. Catalano RA, Nelson LB, Calhoun JH, Schatz NJ, Harley RD. Persistent strabismus presenting after cataract surgery. Ophthalmology 1987;94:491-4.

5. Antoszyk AN, Buckley EG. Contralateral decreased visual acuity and extra-ocular muscle palsies following retrobulbar anesthesia. Ophthalmology 1986;93:462-5.

6. Ong-Tone L, Pearce WG. Inferior rectus muscle restriction after retrobulbar anesthesia for cataract extraction. Can J Ophthalmol 1989;24:162-5.

7. Hamed LM, Mancuso A. Inferior rectus muscle contracture syndrome after retrobulbar anesthesia. Ophthalmology 1991;98:1506-12.

8. Rainin EA, Carlson BM. Postoperative diplopia and ptosis: a clinical hypothesis based on the myotoxicity of local anesthetics. Arch Ophthalmol 1985:103:1337-9.

9. Chapman JM, Abdelatif OM, Cheeks L, Green K. Subconjunctival gentamicin induction of extraocular toxic muscle myopathy. Ophthalmic Res 1992;24:189-96.

10. Hamed LM, Lingua RW. The diagnosis and management of diplopia after cataract surgery. Semin Ophthalmol 1988;3:197-204.

11. Hamed LM, Lingua RW. Thyroid eye disease presenting after cataract surgery. J Pediatr Ophthalmol Strabismus 1990;27:10-5.

12. Hamed LM. Strabismus presenting after cataract surgery. Ophthalmology 1991;98:247-52.

13. Hamilton SM, Elsas FJ, Dawson TL. A cluster of patients with inferior rectus restriction following local anesthesia for cataract surgery. J Pediatr Ophthalmol Strabismus 1993;30:288-91.

14. Poland PJ, Hiatt RL. The correction of diplopia after cataract extraction. Ann Ophthalmol 1993;25:110-8.

15. Kaplan LJ, Jaffe NS, Clayman HM. Ptosis and cataract surgery: a multivariant computer analysis of a prospective study. Ophthalmology 1985;92:237-42.

16. Kushner BJ. Ocular muscle fibrosis following cataract extraction. Arch Ophthalmol 1988;106:18-9.

17. Burns CL, Seigel LA. Inferior rectus recession for vertical tropia after cataract extraction. Ophthalmology 1988;95:1120-4.

18. Grimmett MR, Lambert SR. Superior rectus muscle overaction after cataract extraction. Am J Ophthalmol 1992;1 14:72-80.

19. Brent P. Cataract patients: pre-operative assessment for fusion potential. Am Orthopt J 1986;36:135-9.

20. Esswein MB, von Noorden GK. Paresis of a vertical rectus muscle after cataract extraction. Am J Ophthalmol 1993:116:424-30 